\title{
Generic Ergodic Capacity Bounds for Fixed-Gain AF Dual-Hop Relaying Systems
}

\author{
Caijun Zhong, Member, IEEE, Michail Matthaiou, Member, IEEE, George K. Karagiannidis, Senior Member, IEEE, \\ and Tharmalingam Ratnarajah, Senior Member, IEEE
}

\begin{abstract}
This paper elaborates on the ergodic capacity of fixed-gain amplify-and-forward (AF) dual-hop systems, which have recently attracted considerable research and industry interest. In particular, two novel capacity bounds that allow for fast and efficient computation and apply for nonidentically distributed hops are derived. More importantly, they are generic since they apply to a wide range of popular fading channel models. Specifically, the proposed upper bound applies to Nakagami- $m$, Weibull, and generalized- $K$ fading channels, whereas the proposed lower bound is more general and applies to Rician fading channels. Moreover, it is explicitly demonstrated that the proposed lower and upper bounds become asymptotically exact in the high signalto-noise ratio (SNR) regime. Based on our analytical expressions and numerical results, we gain valuable insights into the impact of model parameters on the capacity of fixed-gain AF dual-hop relaying systems.
\end{abstract}

Index Terms-Amplify-and-forward (AF) relaying, dual-hop transmission, ergodic capacity, fading channels.

\section{INTRODUCTION}

D UAL-HOP relaying systems, where an intermediate relay node helps to forward the source signal to the intended destination node, have received enormous interest due to their ability to significantly improve the throughput and reliability of communication systems, as well as to provide wider coverage

Manuscript received April 17, 2011; revised July 15, 2011 and August 19, 2011; accepted August 21, 2011. Date of publication September 8, 2011; date of current version October 20, 2011. This work was supported in part by the Engineering and Physical Science Research Council (EPSRC) under Grant EP/G026092/1. The work of M. Matthaiou was supported in part by the Swedish Governmental Agency for Innovation Systems (VINNOVA) within the VINN Excellence Center Chase. The work of T. Ratnarajah was supported by the Future and Emerging Technologies (FET) Program within the Seventh Framework Program for Research of the European Commission under FETOpen Grant CROWN-233843. The review of this paper was coordinated by Prof. S. Muhaidat.

C. Zhong is with Institute of Information and Communication Engineering, Zhejiang University, Hangzhou 310027, China (e-mail: caijun.zhong@ ieee.org).

M. Matthaiou is with the Department of Signals and Systems, Chalmers University of Technology, 41296 Gothenburg, Sweden (e-mail: michail.matthaiou@chalmers.se).

G. K. Karagiannidis is with the Department of Electrical and Computer Engineering, Aristotle University of Thessaloniki, Thessaloniki 54 124, Greece (e-mail: geokarag@auth.gr).

T. Ratnarajah is with the Institute of Electronics, Communications and Information Technology, Queen's University Belfast, BT3 9DT Belfast, U.K. (e-mail: t.ratnarajah@ecit.qub.ac.uk).

Color versions of one or more of the figures in this paper are available online at http://ieeexplore.iee.org.

Digital Object Identifier 10.1109/TVT.2011.2167362 to operators [1]-[3]. In this context, amplify-and-forward (AF) relaying systems, where the relay node amplifies the received signal and retransmits it to the destination, have low hardware requirements and easy deployment and, therefore, are elegant from a practical viewpoint. Generally, depending on the availability of channel state information (CSI) at the relay node, AF dual-hop relaying systems can further be classified into two categories, i.e., variable-gain relaying [2] and fixed-gain relaying [3], with the latter being particularly useful for practical applications thanks to their low implementation complexity.

Understanding the fundamental capacity limits of AF dualhop systems in various practical propagation environments has been an active area of research. For Rayleigh fading channels, Farhadi and Beaulieu [4] proposed an ergodic capacity upper bound for dual-hop systems with different relay gains based on Jensen's inequality. This approach was later extended in [5] by deriving an additional upper bound, based on the harmonic-geometric means inequality, assuming CSI is only known at the destination. The main disadvantage of both bounds is that they are not sufficiently tight, particularly when the hops are not identically distributed. For this reason, the authors in [5] also presented an exact alternative expression [5, Eq. (17)], which, however, is based on an infinite series of scalar integrals, and as such, its evaluation is tedious and not amenable to further manipulations.

Recently, Waqar et al. [6] presented several tight bounds for fixed-gain dual-hop systems based on Steffensen's, Chebyshev's, and Jensen's inequalities. The presented analysis, however, is valid for Rayleigh fading, and an extension to other types of fading does not seem straightforward. The seminal work of [7] on fixed-gain dual-hop systems under Nakagami$m$ fading and with partial relay selection provided a tight and computationally efficient approximation to the ergodic capacity using a Taylor series expansion; yet, this approximation is not strictly greater or smaller than the exact capacity and, hence, cannot serve as an upper/lower bound.

In parallel, Ikki and Ahmed [8] presented an ergodic capacity upper bound, which originates from [9], for variable-gain dualhop systems in Weibull fading channels, whereas Wu et al. [10] obtained an ergodic capacity upper bound for fixed-gain dual-hop systems in generalized- $K$ fading channels based on Jensen's inequality. Finally, Waqar et al. [11] proposed a general framework for analyzing the ergodic capacity of variablegain multihop relaying systems, although the presented results either apply for identically distributed fading distributions (e.g., Nakagami- $m$ ) or rely on the classical moment-based approach of [12]. 
While these prior works have significantly improved our knowledge on the ergodic capacity characterization of dualhop relaying systems, a general capacity framework, which will encompass the most important fading channels, seems to be missing from the literature. In this light, we have tried to bridge this gap by analytically investigating the ergodic capacity of dual-hop systems for several fading models.

In particular, the contributions of this paper can now be summarized as follows.

1) We first propose two novel bounding techniques for the ergodic capacity of fixed-gain dual-hop systems. The proposed capacity bounds (one lower and one upper bound) are generally quite tight and become asymptotically exact in the high signal-to-noise ratio (SNR) regime. We then particularize these general bounds to the most popular and important fading models, namely, Nakagami- $m$, Weibull, Rician, and generalized- $K$ fading channels. For these fading models, some new analytical expressions are derived, which offer rather useful insights into the implications of the model parameters on the performance of dual-hop relaying systems. We note that all the presented results can easily be evaluated and efficiently programmed. More importantly, they are applicable for nonidentically distributed hops, with different fading characteristics.

2) In addition, we devise a new relationship between the ergodic capacity and the first negative moment of the instantaneous SNR of each hop. Since the negative moment does not always exist, we establish a straightforward condition for its existence that is only dependent on the fading parameters.

The rest of this paper is organized as follows: Section II introduces the system model that is used throughout this paper. Section III presents the generic lower and upper bounds on the ergodic capacity of the dual-hop relaying systems, which are then particularized to specific fading channel models in Section IV. Finally, Section V concludes this paper.

\section{System ModeL}

Let us consider a dual-hop relaying system with one source, relay, and destination node; in this case, the end-to-end input and output relationship can succinctly be expressed as [2], [3]

$$
y=h_{2} G\left(h_{1} x+n_{1}\right)+n_{2}
$$

where $x$ denotes the source symbol with $\mathrm{E}\left\{x x^{*}\right\}=P_{1}$, whereas $(\cdot)^{*}$ and $\mathrm{E}\{\cdot\}$ denote complex conjugate and expectation, respectively. The term $h_{1}$ represents the complex random fading coefficient of the source-relay link, whereas $h_{2}$ is the complex random fading coefficient of the relay-destination link. In addition, $n_{1}$ and $n_{2}$ are the additive Gaussian noises at the relay and destination nodes with power $N_{1}$ and $N_{2}$, respectively. Note that the fixed relaying gain can be expressed as $G=\sqrt{P_{2} /\left(\mathrm{E}\left\{\left|h_{1}\right|^{2}\right\} P_{1}+N_{1}\right)}$, where $P_{2}$ is the power of the transmitted signal at the output of the relay. Hence, the end-toend SNR becomes [3]

$$
\rho=\frac{\rho_{1} \rho_{2}}{c+\rho_{2}}
$$

where $\rho_{1}=P_{1}\left|h_{1}\right|^{2} / N_{1}, \rho_{2}=P_{2}\left|h_{2}\right|^{2} / N_{2}$, and $c=P_{2} / G^{2} N_{1}$. Having these definitions in hand, the ergodic capacity of the system reads as

$$
C=\frac{1}{2} \mathrm{E}\left\{\log _{2}(1+\rho)\right\}=\frac{1}{2} \mathrm{E}\left\{\log _{2}\left(1+\frac{\rho_{1} \rho_{2}}{c+\rho_{2}}\right)\right\}
$$

where the factor $1 / 2$ accounts for the fact that the entire communication occupies two time slots. In general, a closedform evaluation of the ergodic capacity in (3) is a challenging mathematical problem due to the presence of the nonlinear log function. On this basis, we hereafter seek to lower and upper bound (3) using some tractable statistical tools.

\section{Generic CAPACITy Bounds}

In this section, we propose two general and tight lower and upper bounds on the ergodic capacity of fixed-gain AF dualhop systems in arbitrary fading channels. We start with the following lower bound.

\section{A. Lower Bound}

Theorem 1: The ergodic capacity of fixed-gain AF dual-hop systems is lower bounded by

$C_{l}=\frac{1}{2} \log _{2}\left(1+\exp \left(\mathrm{E}\left\{\ln \rho_{1}\right\}+\mathrm{E}\left\{\ln \rho_{2}\right\}-\mathrm{E}\left\{\ln \left(c+\rho_{2}\right)\right\}\right)\right)$.

Proof: The proof follows by expressing the ergodic capacity as $(1 / 2) \mathrm{E}\left\{\log _{2}\left(1+\exp \left(\ln \left(\rho_{1} \rho_{2} / c+\rho_{2}\right)\right)\right)\right\}$ and utilizing the fact that $\log _{2}(1+a \exp (x))$ is convex in $x$ for $a>0$; the desired result can be obtained with some simple algebraic manipulations after applying Jensen's inequality.

\section{B. Upper Bound}

To obtain an ergodic capacity upper bound, one straightforward approach is to exploit the fact that $\log _{2} x$ is a concave function with respect to $x$ and, thereafter, apply Jensen's inequality on (3). By doing so, we end up with the following upper bound:

$$
C_{u 1}=\frac{1}{2} \log _{2}\left(1+\mathrm{E}\left\{\rho_{1}\right\} \mathrm{E}\left\{\frac{\rho_{2}}{c+\rho_{2}}\right\}\right) .
$$

We note that, in the context of dual-hop relaying systems, this bounding technique was initially used in [4] and later adopted in [10] when studying the ergodic capacity of fixed-gain AF dualhop systems in Rayleigh and generalized- $K$ fading channels, respectively. However, the main disadvantage of such bound is its inherently poor performance in the high SNR regime. Motivated by this critical limitation and after a simple transformation, we derive a novel capacity upper bound that becomes exact in the high SNR regime.

Theorem 2: The ergodic capacity of fixed-gain AF dual-hop systems is upper bounded by

$$
\begin{aligned}
& C_{u 2}=\frac{1}{2} \log _{2}\left(1+c \mathrm{E}\left\{\rho_{1}^{-1}\right\} \mathrm{E}\left\{\rho_{2}{ }^{-1}\right\}+\mathrm{E}\left\{\rho_{1}^{-1}\right\}\right) \\
&+\frac{1}{2} \mathrm{E}\left\{\log _{2} \rho_{1}+\log _{2} \rho_{2}-\log _{2}\left(c+\rho_{2}\right)\right\} .
\end{aligned}
$$


Proof: The ergodic capacity can alternatively be expressed as

$$
C=\frac{1}{2} \mathrm{E}\left\{\log _{2}\left(1+\frac{c+\rho_{2}}{\rho_{1} \rho_{2}}\right)\right\}+\frac{1}{2} \mathrm{E}\left\{\log _{2}\left(\frac{\rho_{1} \rho_{2}}{c+\rho_{2}}\right)\right\} .
$$

Then, applying the Jensen's inequality on the first term yields the desired result.

From (6), it is evident that the evaluation of $C_{u 2}$ requires the existence of the first negative moment of a random variable. In this sense, the following lemma will be particularly useful.

Lemma 1[13]: For a continuous random variable $X$ with probability density function (pdf) $f_{X}(x)$, its first negative moment $\mathrm{E}\left\{x^{-1}\right\}$ does not exist if it has a positive mass at $X=0$, i.e., $f_{X}(0)>0$.

It is also worth mentioning that, in the asymptotically high SNR regime, i.e., $\rho_{1}$ and $\rho_{2}$ are large, both lower and upper bounds $C_{l}$ and $C_{u 2}$ become exact and equal to

$$
C^{\infty}=C_{l}^{\infty}=C_{u 2}^{\infty}=\frac{1}{2} \mathrm{E}\left\{\log _{2}\left(\frac{\rho_{1} \rho_{2}}{c+\rho_{2}}\right)\right\} .
$$

\section{CAPacity Bounds For Fading Channels}

In this section, we particularize the general capacity bounds proposed in the previous section to the most important fading channel models, including Nakagami- $m$, Weibull, Rician, and generalized- $K$ fading channels.

\section{A. Nakagami-m Fading Channels}

The Nakagami- $m$ distribution [14] is a general fading model that includes the one-sided Gaussian distribution $(m=1 / 2)$ and the Rayleigh distribution $(m=1)$ as special cases, and it can also accurately approximate the Nakagami- $q$ (Hoyt) distribution. Most importantly, it often yields the best fit with real-time data in various land-mobile [15] and indoor-mobile multipath [16], [17] propagation environments. For Nakagami- $m$ fading channels, we assume that $\left|h_{1}\right|$ and $\left|h_{2}\right|$ are independent and nonidentically distributed Nakagami- $m$ random variables with parameters $m_{1}, \Omega_{1}$, and $m_{2}, \Omega_{2}$, respectively. The pdf of $\left|h_{i}\right|^{2}(i=1,2)$ is then given by

$$
p(x)=\frac{1}{\Gamma\left(m_{i}\right)}\left(\frac{m_{i}}{\Omega_{i}}\right)^{m_{i}} x^{m_{i}-1} \exp \left(-\frac{m_{i}}{\Omega_{i}} x\right), \quad m_{i} \geq 0.5
$$

where $\Gamma(x)=\int_{0}^{\infty} t^{x-1} \exp (-t) d t$ denotes the well-known Gamma function [18, Eq. (8.310.1)]. It is also worth mentioning that $\Omega_{i}$ is the average power of the fading channels and is defined as $\Omega_{i}=\mathrm{E}\left\{\left|h_{i}\right|^{2}\right\}, i=1,2$.

We first present the following closed-form lower bound on the ergodic capacity.

Proposition 1: The ergodic capacity of fixed-gain AF dual-hop systems in Nakagami- $m$ fading channels is lower bounded by

$$
\begin{aligned}
C_{l}=\frac{1}{2} \log _{2} & \left(1+\frac{P_{1} P_{2} \Omega_{1} \Omega_{2}}{m_{1} m_{2} N_{1} N_{2} c} \exp \left(\psi\left(m_{1}\right)+\psi\left(m_{2}\right)\right.\right. \\
& \left.\left.-\frac{1}{\Gamma\left(m_{2}\right)} G_{3,2}^{1,3}\left[\frac{P_{2} \Omega_{2}}{N_{2} c m_{2}} \mid \begin{array}{c}
1-m_{2}, 1,1 \\
1,0
\end{array}\right]\right)\right)
\end{aligned}
$$

where $\psi(x)$ is the digamma function [18, Eq. (8.360.1)], and $G_{p, q}^{m, n}\left[x,\left.\right|_{\beta_{1}, \ldots, \beta_{q}} ^{\alpha_{1}, \ldots, \alpha_{p}}\right]$ denotes the Meijer's $G$ function [18, Eq. (9.301)].

\section{Proof: See Appendix A.}

Proposition 1 provides a general expression of the capacity lower bound, which is valid for arbitrary fading parameters. For the special case when $m_{2}$ is an integer, we have the following alternative expression.

Corollary 1: When $m_{2}$ is a positive integer, (8) simplifies to

$$
\begin{array}{r}
C_{l}=\frac{1}{2} \log _{2}\left(1+\frac{P_{1} P_{2} \Omega_{1} \Omega_{2}}{m_{1} m_{2} N_{1} N_{2} c} \exp \left(\psi\left(m_{1}\right)+\psi\left(m_{2}\right)\right.\right. \\
\left.\left.-\exp \left(\frac{c m_{2} N_{2}}{\Omega_{2} P_{2}}\right) \sum_{k=1}^{m_{2}} E_{k}\left(\frac{c m_{2} N_{2}}{\Omega_{2} P_{2}}\right)\right)\right)
\end{array}
$$

where $E_{n}(x)=\int_{1}^{\infty}\left(e^{-x t} / t^{n}\right) d t$ is the exponential integral function of order $n$, for $n=0,1,2, \ldots$, and $\operatorname{Re}(x)>0$ [19, Eq. (5.1.4)].

Proof: The result follows from [20, Eq. (47)]

$$
\mathrm{E}\left\{\ln \left(c+\rho_{i}\right)\right\}=\ln c+\exp \left(\frac{c m_{i} N_{i}}{\Omega_{i} P_{i}}\right) \sum_{k=1}^{m_{i}} E_{k}\left(\frac{c m_{i} N_{i}}{\Omega_{i} P_{i}}\right)
$$

and some basic algebraic manipulations.

As a next step, we consider the upper bounds on the ergodic capacity. For general noninteger $m_{2}$, the first upper bound can be obtained via the moment expression of the end-to-end SNR [21, Eq. (10)]. Here, we present an alternative simplified expression for the case of $m_{2}$ being an integer.

Proposition 2: When $m_{2}$ is a positive integer, the ergodic capacity of fixed-gain AF dual-hop systems in Nakagami- $m$ fading channels is upper bounded by

$$
\begin{aligned}
C_{u 1}=\frac{1}{2} \log _{2} & \left(1+\frac{P_{1} \Omega_{1} m_{2}}{N_{1}}\right. \\
& \left.\times \exp \left(\frac{m_{2} N_{2} c}{P_{2} \Omega_{2}}\right) E_{m_{2}+1}\left(\frac{m_{2} N_{2} c}{P_{2} \Omega_{2}}\right)\right) .
\end{aligned}
$$

Proof: Since $\mathrm{E}\left\{\left|h_{i}\right|^{2}\right\}=\Omega_{i}$, the key task is to evaluate $\mathrm{E}\left\{\left|h_{i}\right|^{2} /\left(\left|h_{i}\right|^{2}+a\right)\right\}$, which is given by

$$
\begin{aligned}
\mathrm{E}\left\{\frac{\left|h_{i}\right|^{2}}{\left|h_{i}\right|^{2}+a}\right\}=\frac{1}{\Gamma\left(m_{i}\right)} & \left(\frac{m_{i}}{\Omega_{i}}\right)^{m_{i}} \\
& \times \underbrace{\int_{0}^{\infty} \frac{x^{m_{i}}}{x+a} \exp \left(-\frac{m_{i}}{\Omega_{i}} x\right) d x}_{\mathcal{I}_{1}} .
\end{aligned}
$$

The integral $\mathcal{I}_{1}$ can be evaluated as

$$
\begin{aligned}
\mathcal{I}_{1} & =a^{m_{i}} \exp \left(\frac{m_{i} a}{\Omega_{i}}\right) \Gamma\left(m_{i}+1\right) \Gamma\left(-m_{i}, \frac{m_{i} a}{\Omega_{i}}\right) \\
& =\exp \left(\frac{m_{i} a}{\Omega_{i}}\right)\left(\frac{m_{i}}{\Omega_{i}}\right)^{-m_{i}} E_{m_{i}+1}\left(\frac{m_{i} a}{\Omega_{i}}\right) \Gamma\left(m_{i}+1\right)
\end{aligned}
$$


where $\Gamma(n, x)=\int_{x}^{\infty} x^{n-1} e^{-x} d x$ is the upper incomplete gamma function [18, Eq. (8.350.2)]. Note that (12) is obtained with the aid of [18, Eq. (3.383.10)], whereas (13) follows from the identity [19, Eq. (5.1.45)]. Substituting (13) into (11), we have

$$
\mathrm{E}\left\{\frac{\left|h_{i}\right|^{2}}{\left|h_{i}\right|^{2}+a}\right\}=m_{i} \exp \left(\frac{m_{i} a}{\Omega_{i}}\right) E_{m_{i}+1}\left(\frac{m_{i} a}{\Omega_{i}}\right)
$$

and this concludes the proof.

On a similar basis, the second upper bound is analytically given by the following proposition.

Proposition 3: The ergodic capacity of fixed-gain AF dualhop systems in Nakagami- $m$ fading channels is upper bounded by $C_{u 2}$, as in (15), shown at the bottom of the page.

Proof: The key of the proof is to obtain the first negative moment of $\left|h_{i}\right|^{2}$, which can be computed as

$$
\begin{aligned}
\mathrm{E}\left\{\frac{1}{\left|h_{i}\right|^{2}}\right\} & =\frac{1}{\Gamma\left(m_{i}\right)}\left(\frac{m_{i}}{\Omega_{i}}\right)^{m_{i}} \int_{0}^{\infty} x^{m_{i}-2} \exp \left(-\frac{m_{i}}{\Omega_{i}} x\right) d x \\
& =\frac{m_{i}}{\left(m_{i}-1\right) \Omega_{i}}
\end{aligned}
$$

where we have used [18, Eq. (3.381.5)] to solve the corresponding integral. Substituting (16), (35), and (38) into (6), we can obtain (15) after some basic algebra.

Note that the upper bound (15) is valid for arbitrary $m_{2}$. For the special case where $m_{2}$ is a positive integer, we have the following alternative expression.

Corollary 2: When $m_{2}$ is a positive integer, the ergodic capacity upper bound (15) reduces to (17), shown at the bottom of the page.

Proof: The proof follows easily by combining the results presented in Corollary 1 and Proposition 3.

It is also worth pointing out that, according to Lemma 1 , the first negative moment exists only if $m_{i}>1, i=1,2$; thus, $C_{u 2}$ does not exist if at least one of the hops is Rayleigh faded.

Fig. 1 illustrates the performance of the derived upper and lower bounds in Nakagami- $m$ fading channels. We observe that, in general, the second upper bound $C_{u 2}$ is tighter

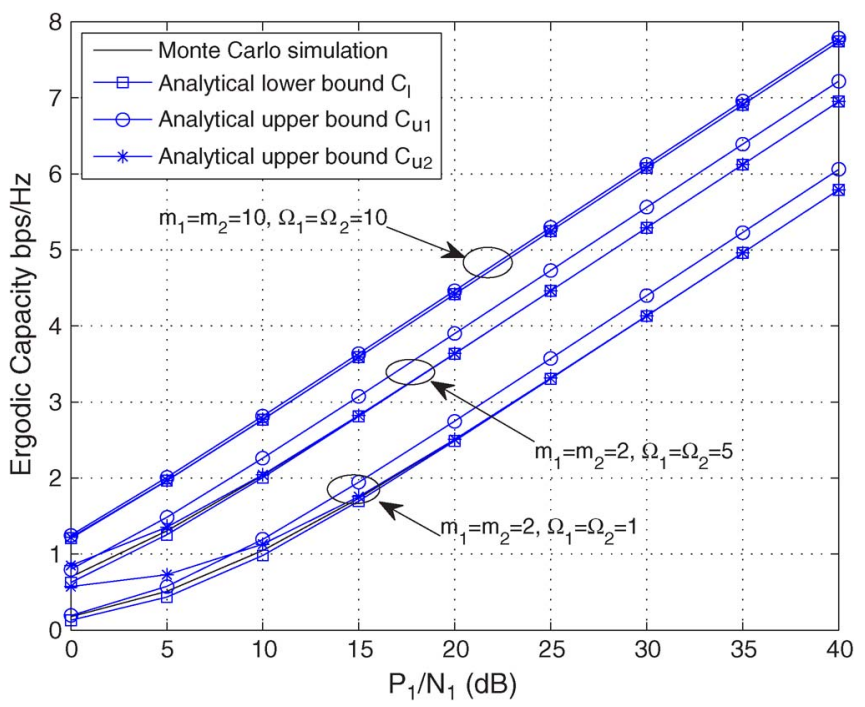

Fig. 1. Ergodic capacity of fixed-gain AF dual-hop systems in Nakagami$m$ fading channels: simulation results, analytical upper and lower bounds. $P_{1} / N_{1}=P_{2} / N_{2}$.

than the first upper bound $C_{u 1}$ in the high SNR regime. Moreover, the tightness of the second upper bound $C_{u 2}$ improves when the channel conditions become better, i.e., the channel gains $\Omega_{i}$ or the fading parameters $m_{i}$ become large. On the other hand, the lower bound $C_{l}$ remains tight across the entire SNR range; in fact, in the high SNR regime, it overlaps with the second upper bound $C_{u 2}$, thereby validating that these two bounds are asymptotically exact at high SNRs. Generally speaking, the effect of $m_{i}$ on the capacity becomes less pronounced as $m_{i}$ gets larger (i.e., the relative difference between the capacity curves gets smaller). This is consistent with the results in [7] and [21].

\section{B. Weibull Fading Channels}

The Weibull fading model has recently attracted considerable research interest thanks to its ability to yield good fit to experimental fading channel measurements for both indoor [22] and outdoor environments [23]. In this case, we assume that $\left|h_{1}\right|$ and $\left|h_{2}\right|$ are independent and nonidentically distributed

$$
\begin{aligned}
C_{u 2}=\frac{1}{2} \log _{2}\left(1+\frac{N_{1} N_{2} m_{1} m_{2} c}{P_{1} P_{2}\left(m_{1}-1\right)\left(m_{2}-1\right) \Omega_{1} \Omega_{2}}+\frac{N_{1} m_{1}}{P_{1}\left(m_{1}-1\right) \Omega_{1}}\right) & \\
& \quad+\frac{1}{2 \ln 2}\left(\ln \left(\frac{P_{1} P_{2} \Omega_{1} \Omega_{2}}{m_{1} m_{2} N_{1} N_{2} c}\right)+\psi\left(m_{1}\right)+\psi\left(m_{2}\right)-\frac{1}{\Gamma\left(m_{2}\right)} G_{3,2}^{1,3}\left[\frac{P_{2} \Omega_{2}}{N_{2} c m_{2}} \mid \begin{array}{c}
1-m_{2}, 1,1 \\
1,0
\end{array}\right]\right)
\end{aligned}
$$

$$
\begin{aligned}
C_{u 2}=\frac{1}{2} \log _{2}\left(1+\frac{N_{1} N_{2} m_{1} m_{2} c}{P_{1} P_{2}\left(m_{1}-1\right)\left(m_{2}-1\right) \Omega_{1} \Omega_{2}}+\frac{N_{1} m_{1}}{P_{1}\left(m_{1}-1\right) \Omega_{1}}\right) & \\
+ & \frac{1}{2 \ln 2}\left(\ln \left(\frac{P_{1} P_{2} \Omega_{1} \Omega_{2}}{m_{1} m_{2} N_{1} N_{2} c}\right)+\psi\left(m_{1}\right)+\psi\left(m_{2}\right)-\exp \left(\frac{c m_{2} N_{2}}{\Omega_{2} P_{2}}\right) \sum_{k=1}^{m_{2}} E_{k}\left(\frac{c m_{2} N_{2}}{\Omega_{2} P_{2}}\right)\right)
\end{aligned}
$$


Weibull random variables with parameters $m_{1}, \Omega_{1}$, and $m_{2}, \Omega_{2}$, respectively. Hence, the pdf of $\left|h_{i}\right|^{2}(i=1,2)$ is given by

$$
p(x)=\frac{m_{i}}{2 \Omega_{i}} x^{\frac{m_{i}}{2}-1} \exp \left(-\frac{1}{\Omega_{i}} x^{\frac{m_{i}}{2}}\right) .
$$

Proposition 4: The ergodic capacity of fixed-gain AF dualhop systems in Weibull fading channels is lower bounded by

$C_{l}=\frac{1}{2} \log _{2}\left(1+\frac{P_{1} P_{2} \Omega_{1}^{\frac{2}{m_{1}}} \Omega_{2}^{\frac{2}{m_{2}}}}{N_{1} N_{2} c} \exp \left(-\frac{2 \gamma}{m_{1}}-\frac{2 \gamma}{m_{2}}-A_{1}\right)\right)$

where $\gamma=0.577216$ is the Euler constant, and $A_{1}$ is given by

$$
\begin{aligned}
A_{1} & =\frac{m_{2} \sqrt{k}}{2 \Omega_{2} l(2 \pi)^{l+\frac{k}{2}-\frac{3}{2}}}\left(\frac{P_{2}}{c N_{2}}\right)^{-\frac{m_{2}}{2}} \\
& \times G_{2 l, k+2 l}^{k+2 l, l}\left(\frac{\left(c N_{2}\right)^{l}}{\left(k \Omega_{2}\right)^{k} P_{2}^{l}} \mid \begin{array}{l}
\Delta\left(l,-\frac{m_{2}}{2}\right), \Delta\left(l, 1-\frac{m_{2}}{2}\right) \\
\Delta(k, 0), \Delta\left(l,-\frac{m_{2}}{2}\right), \Delta\left(l,-\frac{m_{2}}{2}\right)
\end{array}\right)
\end{aligned}
$$

where $\Delta(n, \epsilon) \triangleq(\epsilon / n),(\epsilon+1 / n), \ldots,(\epsilon+n-1 / n)$, with $\epsilon$ as an arbitrary real value and $n$ as a positive integer. Moreover, $(l / k)=\left(m_{2} / 2\right)$, where $l$ and $k$ are positive integers.

Proof: The term $A_{1}=\mathrm{E}\left\{\ln \left(1+\rho_{2} / c\right)\right\}$ has already been derived in [24, Eq. (17)]. As such, it suffices to compute $\mathrm{E}\left\{\ln \rho_{i}\right\}$, which can be evaluated as follows:

$$
\begin{aligned}
\mathrm{E}\left\{\ln \rho_{i}\right\}= & \ln \frac{P_{i}}{N_{i}} \\
& +\frac{m_{i}}{2 \Omega_{i}} \underbrace{\int_{0}^{\infty} x^{\frac{m_{i}}{2}-1} \exp \left(-\frac{1}{\Omega_{i}} x^{\frac{m_{i}}{2}}\right) \ln x d x}_{\mathcal{I}_{2}} .
\end{aligned}
$$

Making a change of variable $t=x^{m_{i} / 2}$, the integral can be computed as

$$
\mathcal{I}_{2}=\left(\frac{2}{m_{i}}\right)^{2} \int_{0}^{\infty} \exp \left(-\frac{t}{\Omega_{i}}\right) \ln t d t .
$$

Invoking the integration relationship [18, Eq. (4.352.1)]

$$
\mathrm{E}\left\{\ln \rho_{i}\right\}=\ln \frac{P_{i}}{N_{i}}-\frac{2}{m_{2}}\left(\gamma-\ln \Omega_{i}\right) .
$$

Combining (22) with (4), we obtain the desired result.

Proposition 5: The ergodic capacity of fixed-gain AF dualhop systems in Weibull fading channels is upper bounded by

$$
\begin{array}{r}
C_{u 1}=\frac{1}{2} \log _{2}\left(1+\frac{\sqrt{k} P_{1} \Omega_{1}^{\frac{2}{m_{1}}} \Gamma\left(\frac{2}{m_{1}}\right) m_{2}\left(c N_{2}\right)^{\frac{m_{2}}{2}}}{N_{1} m_{1} P_{2}^{\frac{m_{2}}{2}} \Omega_{2}(2 \pi)^{l+\frac{k}{2}-\frac{3}{2}}}\right. \\
\left.\times G_{l, k+l}^{k+l, l}\left(\frac{\left(c N_{2}\right)^{l}}{P_{2}^{l}\left(k \Omega_{2}\right)^{k}} \begin{array}{l}
\Delta\left(l,-\frac{m_{2}}{2}\right) \\
\Delta(k, 0), \Delta\left(l,-\frac{m_{2}}{2}\right)
\end{array}\right)\right) .
\end{array}
$$

Proof: See Appendix B.

Proposition 6: The ergodic capacity of fixed-gain AF dualhop systems in Weibull fading channels is upper bounded by

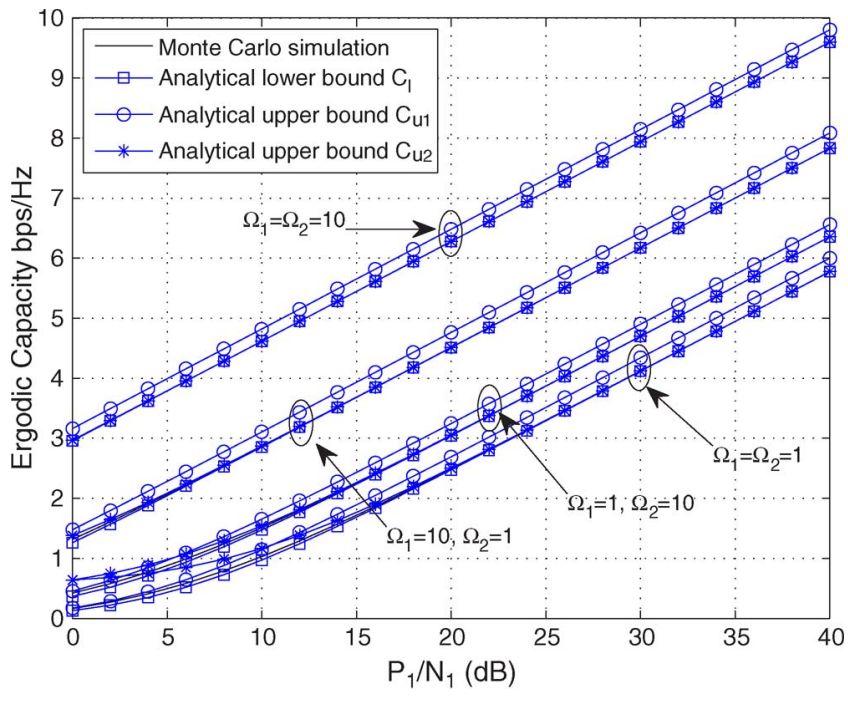

Fig. 2. Ergodic capacity of fixed-gain AF dual-hop systems in Weibull fading channels: simulation results, analytical upper and lower bounds. $P_{1} / N_{1}=$ $P_{2} / N_{2}$.

$C_{u 2}$ as

$$
\begin{aligned}
C_{u 2}= & \frac{1}{2} \log _{2}\left(1+\frac{N_{1} N_{2} c}{P_{1} P_{2}} \Omega_{1}^{-\frac{2}{m_{1}}} \Gamma\left(1-\frac{2}{m_{1}}\right)\right. \\
& \left.\times \Omega_{2}^{-\frac{2}{m_{2}}} \Gamma\left(1-\frac{2}{m_{2}}\right)+\frac{N_{1}}{P_{1}} \Omega_{1}^{-\frac{2}{m_{1}}} \Gamma\left(1-\frac{2}{m_{1}}\right)\right) \\
& +\frac{1}{2 \ln 2}\left(\ln \left(\frac{P_{1} P_{2} \Omega_{1}^{\frac{2}{m_{1}}} \Omega_{2}^{\frac{2}{m_{2}}}}{N_{1} N_{2} c}\right)-\frac{2 \gamma}{m_{1}}-\frac{2 \gamma}{m_{2}}-A_{1}\right) .
\end{aligned}
$$

Proof: The key of the proof is to obtain the first negative moment of $\left|h_{i}\right|^{2}$, which can easily be computed as

$$
\begin{aligned}
\mathrm{E}\left\{\frac{1}{\left|h_{i}\right|^{2}}\right\} & =\frac{m_{i}}{2 \Omega_{i}} \int_{0}^{\infty} x^{\frac{m_{i}}{2}-2} \exp \left(-\frac{1}{\Omega_{i}} x^{\frac{m_{i}}{2}}\right) d x \\
& =\Omega_{i}^{-\frac{2}{m_{i}}} \Gamma\left(1-\frac{2}{m_{i}}\right)
\end{aligned}
$$

where, once more, we have used [18, Eq. (3.381.5)] to solve the corresponding integral. Substituting (22) and (25) into (6), we can get (24) after factorization.

The existence of the first negative moment in the Weibull case requires $m_{i}>2, i=1,2$; this implies that $C_{u 2}$ does not exist if at least one of the hops is Rayleigh faded, reflecting those observations that have also been made in the Nakagami$m$ case.

Fig. 2 investigates the performance of the proposed upper and lower bounds over asymmetric Weibull fading channels with $m_{1}=3$ and $m_{2}=6$. Not surprisingly, we see that when the average channel gain of either hop increases, the achievable capacity also increases. Moreover, Fig. 2 demonstrates that the achievable capacity for the $\Omega_{1}=10, \Omega_{2}=1$ case is larger than that of the $\Omega_{1}=1, \Omega_{2}=10$ case, suggesting that the average channel gain of the first hop has a greater impact on 
the system performance compared with the average channel gain of the second hop. In addition, we see that the tightness of the first upper bound $C_{u 1}$ is relatively insensitive to the change of channel gain $\Omega_{i}$, whereas the tightness of the second upper bound $C_{u 2}$ and lower bound $C_{l}$ improves when $\Omega_{i}$ increases.

\section{Rician Fading Channels}

The Rician fading model is suitable when a direct line-ofsight or specular component is present in the wireless channel. In particular, this type of fading is usually observed in the first resolvable line-of-sight path of microcellular urban and suburban land-mobile [25] and picocellular indoor environments [26]. We recall that the Rician distribution spans the range from Rayleigh fading $K=0$ to no fading $K=\infty$, where $K$ denotes the Rician factor. In this case, we assume that $\left|h_{1}\right|$ and $\left|h_{2}\right|$ are independent and nonidentically distributed Rician random variables with parameters $K_{1}, \Omega_{1}$, and $K_{2}, \Omega_{2}$, respectively. Hence, the pdf of $\left|h_{i}\right|^{2}(i=1,2)$ is given by

$$
\begin{aligned}
p(x)=\frac{\left(1+K_{i}\right) e^{-K_{i}}}{\Omega_{i}} \exp ( & \left.-\frac{\left(1+K_{i}\right) x}{\Omega_{i}}\right) \\
& \times I_{0}\left(2 \sqrt{\frac{K_{i}\left(1+K_{i}\right) x}{\Omega_{i}}}\right)
\end{aligned}
$$

where $I_{0}(x)$ is the zeroth-order modified Bessel function of the first kind [18, Eq. (8.405.1)]. We point out that the case of Rician fading is scarcely addressed in the literature due to the presence of the Bessel function, which makes the mathematical analysis tedious. In the following, we present closed-form lower and upper bounds for fixed-gain AF dual-hop systems in Rician fading channels.

Proposition 7: The ergodic capacity of fixed-gain AF dualhop systems in Rician fading channels is lower bounded by $C_{l}$ in (27), shown at the bottom of the page, where $N$ is an arbitrary positive integer, and $\gamma(p, x)=\int_{0}^{x} t^{p-1} e^{-t} d t$ is the lower incomplete gamma function [18, Eq. (8.350.1)].
Proof: See Appendix C.

Proposition 8: The ergodic capacity of dual-hop fixed-gain relaying systems in Rician fading channels is upper bounded by $C_{u 1}$ in (28), shown at the bottom of the page.

Proof: See Appendix D.

The choice of $N$ will affect the tightness of the upper bound $C_{u 1}$ and lower bound $C_{l}$, and generally, a larger $N$ produces a tighter lower or upper bound. Moreover, we notice that the value of $K_{2}$ has a dominating effect on the convergence speed, i.e., how large should $N$ be, such that further increasing $N$ does not produce any perceptible improvement on the tightness of the bound. In general, a smaller $N$ is required for a smaller $K_{2}$, and from our simulations, we find out that, for $K_{2} \leq 10$, the improvement brought by further increasing $N$ beyond 20 becomes negligible.

Proposition 9: For dual-hop fixed-gain relaying systems in Rician fading channels, the upper bound $C_{u 2}$ does not exist.

Proof: From (26), we can easily infer that the pdf mass at $x=0$ is given by $p(0)=\left(\left(1+K_{i}\right) e^{-K_{i}} / \Omega_{i}\right)>0$. Thus, according to Lemma 1, the first negative moment of $\rho_{i}$ does not exist, which, in turn, implies that $C_{u 2}$ does not exist for the Rician case.

Fig. 3 examines the performance of the proposed upper and lower bounds in Rician fading channels. As already mentioned, the choice of $N$ has a significant impact on the tightness of the bounds. In the simulations, we have set $N=1,10,20$ to obtain the curves associated with $K_{2}=0.1,1,10$, respectively (we find out that further increasing $N$ does not produce any noticeable improvement of the bounds). We observe that the ergodic capacity increases when $K_{i}$ becomes large due to the additional power that is captured by the destination node. In addition, the tightness of both upper and lower bounds improves when $K_{i}$ and $\Omega_{i}$ increase.

\section{Generalized K Fading Channels}

This is a generic composite model that emerges when smallscale fading is modeled via the Nakagami- $m$ distribution and large-scale fading via the gamma distribution. This model has

$$
\begin{aligned}
C_{l}= & \frac{1}{2} \log _{2}\left(1+\frac{P_{1} P_{2} \Omega_{1} \Omega_{2} K_{1} K_{2}}{\left(1+K_{1}\right)\left(1+K_{2}\right) N_{1} N_{2} c} \exp \left(E_{1}\left(K_{1}\right)+E_{1}\left(K_{2}\right)-\exp \left(\frac{c\left(1+K_{2}\right) N_{2}}{\Omega_{2} P_{2}}-K_{2}\right) \sum_{j=0}^{N-1} \frac{K_{2}^{j}}{j !}\right.\right. \\
& \left.\left.\times \sum_{k=1}^{j+1} E_{k}\left(\frac{c\left(1+K_{2}\right) N_{2}}{\Omega_{2} P_{2}}\right)-\exp \left(\frac{c\left(1+K_{2}\right) N_{2}}{\Omega_{2} P_{2}}\right) E_{1}\left(\frac{\left(1+K_{2}\right) N_{2}}{\Omega_{2} P_{2}}\right)\left(\frac{K_{2}(N-1) \gamma\left(N-1, K_{2}\right)+\gamma\left(N, K_{2}\right)}{\Gamma(N)}\right)\right)\right)
\end{aligned}
$$

$$
\begin{aligned}
C_{u 1}=\frac{1}{2} \log _{2}\left(1+P_{1} \Omega_{1} N_{1} \exp \left(\frac{\left(1+K_{2}\right) N_{2} c}{P_{2} \Omega_{2}}-K_{2}\right)\left(\sum_{k=0}^{N-1} \frac{(k+1) K_{2}^{k}}{k !} E_{k+2}\left(\frac{\left(1+K_{2}\right) N_{2} c}{P_{2} \Omega_{2}}\right)\right.\right. \\
\left.\left.\quad+E_{N+2}\left(\frac{\left(1+K_{2}\right) N_{2} c}{P_{2} \Omega_{2}}\right) \exp \left(K_{2}\right)\left(\frac{K_{2}(N-1) \gamma\left(N-1, K_{2}\right)+\gamma\left(N, K_{2}\right)}{\Gamma(N)}\right)\right)\right)
\end{aligned}
$$




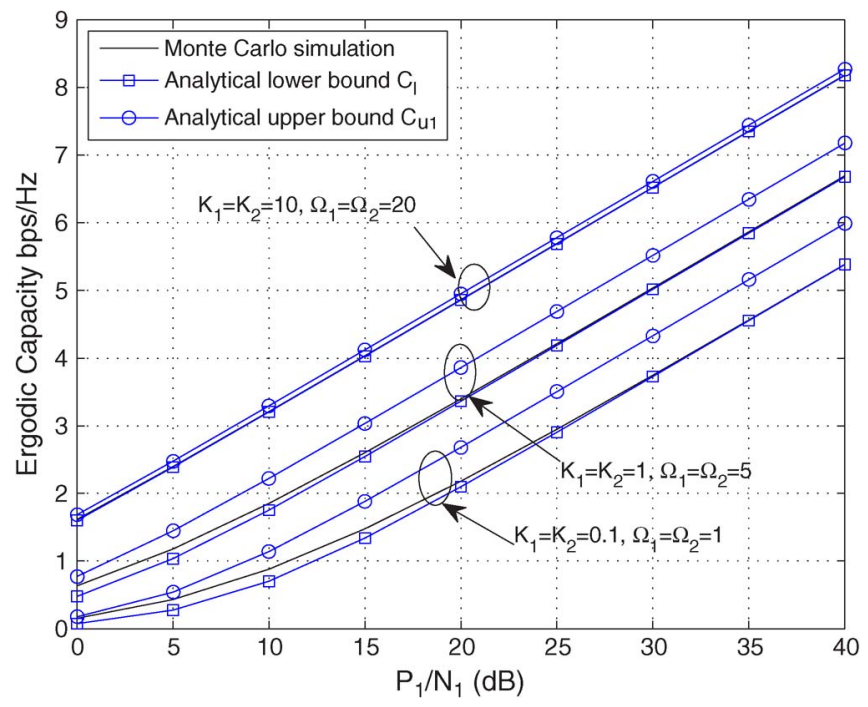

Fig. 3. Ergodic capacity of fixed-gain AF dual-hop systems in Rician fading channels: simulation results, analytical upper and lower bounds. $P_{1} / N_{1}=$ $P_{2} / N_{2}$.

been demonstrated to effectively approximate most of the fading and shadowing effects occurring in wireless channels and also to be analytically friendlier than the classical Nakagami$m /$ lognormal model [27]-[31]. In this case, we assume that $\left|h_{1}\right|$ and $\left|h_{2}\right|$ are independent and nonidentically distributed generalized $K$ random variables with parameters $m_{1}, k_{1}, \Omega_{1}$, and $m_{2}, k_{2}, \Omega_{2}$, respectively. Hence, the pdf of $\left|h_{i}\right|^{2}(i=1,2)$ can be expressed as [28]

$$
p(x)=\frac{2 x^{\frac{k_{i}+m_{i}}{2}-1}}{\Gamma\left(m_{i}\right) \Gamma\left(k_{i}\right)}\left(\frac{m_{i}}{\Omega_{i}}\right)^{\frac{k_{i}+m_{i}}{2}} K_{k_{i}-m_{i}}\left(2 \sqrt{\frac{m_{i}}{\Omega_{i}} x}\right)
$$

where $K_{\nu}(x)$ denotes the $\nu$ th-order modified Bessel function of the second kind [18, Eq. (8.407.1)].

Proposition 10: The ergodic capacity of fixed-gain AF dual-hop systems in generalized- $K$ fading channels is lower bounded by $C_{l}$ in (29), shown at the bottom of the page.

$$
A_{2}=G_{2,4}^{4,1}\left(\frac{m_{2} N_{2} c}{\Omega_{2} P_{2}} \mid \begin{array}{l}
-\frac{k_{2}+m_{2}}{2}, 1-\frac{k_{2}+m_{2}}{2} \\
k_{2}-m_{2} 2, \frac{m_{2}-k_{2}}{2},-k_{2}+m_{2} 2,-\frac{k_{2}+m_{2}}{2}
\end{array}\right) .
$$

Proof: The term $A_{2}=\mathrm{E}\left\{\ln \left(1+\rho_{2} / c\right)\right\}$ can directly be obtained from [28, Eq. (7)]. As such, the key task is to evaluate $\mathrm{E}\left\{\ln \left|h_{i}\right|^{2}\right\}$ or

$$
\begin{aligned}
E\left\{\ln \left|h_{i}\right|^{2}\right\} & =\frac{2}{\Gamma\left(m_{i}\right) \Gamma\left(k_{i}\right)}\left(\frac{m_{i}}{\Omega_{i}}\right)^{\frac{k_{i}+m_{i}}{2}} \\
& \times \int_{0}^{\infty} x^{\frac{k_{i}+m_{i}}{2}-1} K_{k_{i}-m_{i}}\left(2 \sqrt{\frac{m_{i}}{\Omega_{i}} x}\right) \ln x d x .
\end{aligned}
$$

With the help of the integration relationship [32, Eq. (2.16.20.1)], we have $\mathrm{E}\left\{\ln \left|h_{i}\right|^{2}\right\}=\psi\left(k_{i}\right)+\psi\left(m_{i}\right)+$ $\ln \left(\Omega_{i} / m_{i}\right)$.

Now, we turn our attention to the capacity upper bounds. Since the first upper bound $C_{u 1}$ has already been derived in [10], we elaborate on the second upper bound $C_{u 2}$ and propose the following key result, which, to the best of our knowledge, is new.

Proposition 11: The ergodic capacity of fixed-gain AF dualhop systems in generalized $K$ fading channels is upper bounded by $C_{u 2}$ in (31), shown at the bottom of the page.

Proof: The key issue is to obtain the first negative moment of $\left|h_{i}\right|^{2}$, which can be computed as

$$
\begin{aligned}
\mathrm{E}\left\{\frac{1}{\left|h_{i}\right|^{2}}\right\}= & \frac{2}{\Gamma\left(m_{i}\right) \Gamma\left(k_{i}\right)}\left(\frac{m_{i}}{\Omega_{i}}\right)^{k_{i}+m_{i} 2} \\
& \times \int_{0}^{\infty} x^{\frac{k_{i}+m_{i}}{2}-2} K_{k_{i}-m_{i}}\left(2 \sqrt{\frac{m_{i}}{\Omega_{i}} x}\right) d x \\
= & \frac{m_{i}}{\left(m_{i}-1\right)\left(k_{i}-1\right) \Omega_{i}}
\end{aligned}
$$

where from (32) to (33), we have used the integration relationship [18, Eq. (6.561.16)]. Following a similar line of reasoning as in Proposition 10, we can easily work out the desired result in (31).

Note that according to Lemma 1 and the condition for the integration relationship [18, Eq. (6.561.16)] that we have used to evaluate (32), the existence of the first negative moment requires $m_{i}>1$ and $k_{i}>1, i=1,2$.

Fig. 4 compares the lower and upper bounds against the simulation result in generalized $K$ fading channels. Note that the first capacity upper bound $C_{u 1}$ was first presented in [10, Eq. (11)]. Again, we see that the second upper bound $C_{u 2}$ is much tighter than the first upper bound $C_{u 1}$ for moderate/high

$$
C_{l}=\frac{1}{2} \log _{2}\left(1+\frac{P_{1} P_{2} \Omega_{1} \Omega_{2}}{N_{1} N_{2} m_{1} m_{2} c} \exp \left(\psi\left(k_{1}\right)+\psi\left(k_{2}\right)+\psi\left(m_{1}\right)+\psi\left(m_{2}\right)-\frac{A_{2}}{\Gamma\left(k_{2}\right) \Gamma\left(m_{2}\right)}\left(\frac{m_{2} N_{2} c}{\Omega_{2} P_{2}}\right)^{\frac{k_{2}+m_{2}}{2}}\right)\right)
$$

$$
\begin{aligned}
C_{u 2}=\frac{1}{2} \log _{2}(1 & \left.+\frac{N_{1} N_{2} m_{1} m_{2} c}{P_{1} P_{2}\left(m_{1}-1\right)\left(k_{1}-1\right) \Omega_{1}\left(m_{2}-1\right)\left(k_{2}-1\right) \Omega_{2}}+\frac{N_{1} m_{1}}{P_{1}\left(m_{1}-1\right)\left(k_{1}-1\right) \Omega_{1}}\right) \\
& +\frac{1}{2 \ln 2}\left(\ln \frac{P_{1} P_{2} \Omega_{1} \Omega_{2}}{N_{1} N_{2} m_{1} m_{2} c}+\psi\left(k_{1}\right)+\psi\left(k_{2}\right)+\psi\left(m_{1}\right)+\psi\left(m_{2}\right)-\frac{A_{2}}{\Gamma\left(k_{2}\right) \Gamma\left(m_{2}\right)}\left(\frac{m_{2} N_{2} c}{\Omega_{2} P_{2}}\right)^{\frac{k_{2}+m_{2}}{2}}\right)
\end{aligned}
$$




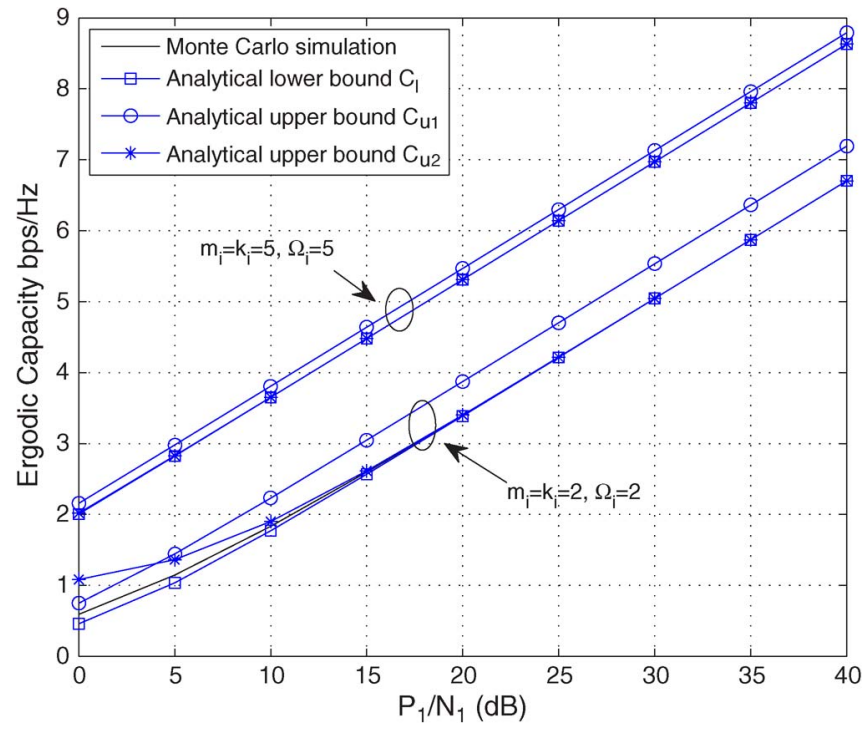

Fig. 4. Ergodic capacity of fixed-gain AF dual-hop systems in generalized$K$ fading channels: simulation results, analytical upper and lower bounds. $P_{1} / N_{1}=P_{2} / N_{2}$.

SNR values, whereas it is in general looser than $C_{u 1}$ in the low SNR regime. This phenomenon is actually quite intuitive from the bounding technique used in these two bounds and the property of Jensen's inequality (i.e., Jensen's inequality is tight if the variance of the random variable is small). In fact, the upper bounds $C_{u 1}$ and $C_{u 2}$ are complementary to each other, i.e., $C_{u 1}$ is tight in the low SNR regime, whereas $C_{u 2}$ is tight in the high SNR regime. Hence, we can potentially construct another upper bound as $\min \left(C_{u 1}, C_{u 2}\right)$, which will be tight across the entire SNR range.

\section{Conclusion}

The main focus of this paper has been on the ergodic capacity of fixed-gain dual-hop systems that have been in the forefront of cooperative communications over the past years. Specifically, we have proposed two novel and generic techniques for bounding the ergodic capacity of these systems. The proposed bounds were also particularized to several fading models of interest, namely, Nakagami- $m$, Weibull, Rician, and generalized- $K$ fading channels, with nonidentically distributed hops, whereas the derived closed-form expressions allow for fast and efficient computation. Moreover, our numerical results demonstrated that the proposed bounds work well under different fading parameters. In fact, in the high SNR regime, they become asymptotically exact.

As a final remark, we highlight the fact that these bounding techniques can easily be extended to the case where the two hop channels experience different types of fading, i.e., asymmetric dual-hop systems [33], as well as to the case with partial relay selection [7].

\section{APPENDIX A}

\section{PROOF OF PROPOSITION 1}

To evaluate the lower bound in (4), we need closed-form expressions for $E\left\{\ln \rho_{i}\right\}$ and $E\left\{\ln \left(c+\rho_{i}\right)\right\}$. To this end, the first term can be computed as

$$
\begin{aligned}
\mathrm{E}\left\{\ln \rho_{i}\right\}=\ln \frac{P_{i}}{N_{i}}+ & \frac{1}{\Gamma\left(m_{i}\right)}\left(\frac{m_{i}}{\Omega_{i}}\right)^{m_{i}} \\
& \times \int_{0}^{\infty} x^{m_{i}-1} \exp \left(-\frac{m_{i}}{\Omega_{i}} x\right) \ln x d x .
\end{aligned}
$$

With the help of the integration relationship [18, Eq. (4.352.1)] and some simple algebraic manipulations, we get

$$
\mathrm{E}\left\{\ln \rho_{i}\right\}=\psi\left(m_{i}\right)+\ln \frac{P_{i} \Omega_{i}}{m_{i} N_{i}} .
$$

Additionally, the second term can be computed as

$$
\begin{aligned}
E\left\{\ln \left(c+\rho_{i}\right)\right\} & =\ln c+\frac{1}{\Gamma\left(m_{i}\right)}\left(\frac{m_{i}}{\Omega_{i}}\right)^{m_{i}} \\
& \times \int_{0}^{\infty} \ln \left(1+\frac{P_{i}}{c N_{i}} x\right) x^{m_{i}-1} \exp \left(-\frac{m_{i}}{\Omega_{i}} x\right) d x .
\end{aligned}
$$

To this end, we express the logarithmic term $\ln (1+\alpha x)$ in terms of Meijer's $G$ function according to [34, Eq. (8.4.6.5)], and we can rewrite (36) according to

$$
\begin{aligned}
& E\left\{\ln \left(c+\rho_{i}\right)\right\} \\
& =\ln c+\frac{1}{\Gamma\left(m_{i}\right)}\left(\frac{m_{i}}{\Omega_{i}}\right)^{m_{i}} \times \int_{0}^{\infty} G_{2,2}^{1,2}\left[\frac{P_{i}}{N_{i} c} x \mid \begin{array}{c}
1,1 \\
1,0
\end{array}\right] x^{m_{i}-1} \\
& \quad \times \exp \left(-\frac{m_{i}}{\Omega_{i}} x\right) d x \\
& =\ln c+\frac{1}{\Gamma\left(m_{i}\right)} G_{3,2}^{1,3}\left[\frac{P_{i} \Omega_{i}}{N_{i} c m_{i}} \mid \begin{array}{c}
1-m_{i}, 1,1 \\
1,0
\end{array}\right]
\end{aligned}
$$

where we have used the integral identity [18, Eq. (7.813.1)]. Hence, the desired result follows immediately by substituting (35) and (38) into (4).

\section{APPENDIX B}

PROOF OF PROPOSITION 5

From (5), it is clear that we have to evaluate $\mathrm{E}\left\{\left|h_{i}\right|^{2}\right\}$ and $\mathrm{E}\left\{\left|h_{i}\right|^{2} /\left(\left|h_{i}\right|^{2}+a\right)\right\}$. The first term can be computed via [18, Eq. (3.381.5)]

$$
\begin{aligned}
\mathrm{E}\left\{\left|h_{i}\right|^{2}\right\} & =\frac{m_{i}}{2 \Omega_{i}} \int_{0}^{\infty} x^{\frac{m_{i}}{2}} \exp \left(-\frac{1}{\Omega_{i}} x^{\frac{m_{i}}{2}}\right) d x \\
& =\frac{2}{m_{i}} \Omega_{i}^{\frac{2}{m_{i}}} \Gamma\left(\frac{2}{m_{i}}\right) .
\end{aligned}
$$

The second term can be rewritten in integral form as

$$
\mathrm{E}\left\{\frac{\left|h_{i}\right|^{2}}{\left|h_{i}\right|^{2}+a}\right\}=\frac{m_{i}}{2 \Omega_{i}} \int_{0}^{\infty} \frac{x^{\frac{m_{i}}{2}}}{x+a} \exp \left(-\frac{1}{\Omega_{i}} x^{\frac{m_{i}}{2}}\right) d x .
$$


Next, we express both $x^{m_{i} / 2} /(x+a)$ and $\exp \left(-\left(1 / \Omega_{i}\right) x^{m_{i} / 2}\right)$ in terms of Meijer's $G$ functions. From [35, p. 54], we have that

$$
\frac{x^{\frac{m_{i}}{2}}}{x+a}=a^{\frac{m_{i}}{2}-1} G_{1,1}^{1,1}\left(\frac{x}{a} \mid \begin{array}{l}
\frac{m_{i}}{m_{i}} \\
\frac{m_{2}}{2}
\end{array}\right)
$$

whereas through [36, Eq. (11)], we get

$$
\exp \left(-\frac{1}{\Omega_{i}} x^{\frac{m_{i}}{2}}\right)=G_{0,1}^{1,0}\left(\left.\frac{x^{\frac{m_{i}}{2}}}{\Omega_{i}}\right|_{0} ^{-}\right) .
$$

Then, using [36, Eq. (14)], the integral can be solved in closed form as follows:

$$
\begin{aligned}
\mathrm{E}\left\{\frac{\left|h_{i}\right|^{2}}{\left|h_{i}\right|^{2}+a}\right\}= & \frac{m_{i}}{2 a \Omega_{i}} a^{\frac{m_{i}}{2}} \frac{\sqrt{k} a}{(2 \pi)^{l+\frac{k}{2}-\frac{3}{2}}} \\
& \times G_{l, k+l}^{k+l, l}\left(\begin{array}{l}
a^{l} \\
\left(k \Omega_{i}\right)^{k}
\end{array} \begin{array}{l}
\Delta\left(l,-\frac{m_{i}}{2}\right) \\
\Delta(k, 0), \Delta\left(l,-\frac{m_{i}}{2}\right)
\end{array}\right) .
\end{aligned}
$$

Combining (39) and (42) with (5), we can readily obtain (23).

\section{APPENDIX C}

\section{PROOF OF PROPOSITION 7}

The key task is to compute $\mathrm{E}\left\{\ln \left|h_{i}\right|^{2}\right\}$ and $\mathrm{E}\left\{\ln \left(c+\rho_{2}\right)\right\}$. The first term can be evaluated after some simple manipulations with the aid of [37, Eq. (404)] as

$$
\mathrm{E}\left\{\ln \left|h_{i}\right|^{2}\right\}=E_{1}\left(K_{i}\right)+\ln \frac{K_{i} \Omega_{i}}{1+K_{i}} .
$$

The second term can be reformulated as follows:

$$
\begin{aligned}
\mathrm{E}\left\{\ln \left(c+\rho_{2}\right)\right\} & =\ln c+\frac{\left(1+K_{2}\right) e^{-K_{2}}}{\Omega_{2}} \sum_{j=0}^{\infty} \frac{\left(K_{2}\left(1+K_{2}\right)\right)^{j}}{\Omega_{2}^{j}(j !)^{2}} \\
& \times \int_{0}^{\infty} \ln \left(1+\frac{P_{2}}{c N_{2}} x\right) \exp \left(-\frac{1+K_{2}}{\Omega_{2}} x\right) x^{j} d x
\end{aligned}
$$

where we have used the infinite series representation of the modified Bessel function of the first kind $I_{0}(x)$ [18, Eq. (8.447.1)]. The integral in the foregoing equation can be solved with a result from [20, Eq. (47)]

$$
\mathrm{E}\left\{\ln \left(c+\rho_{2}\right)\right\}=\ln c+\exp \left(\frac{c\left(1+K_{2}\right) N_{2}}{\Omega_{2} P_{2}}-K_{2}\right) \mathcal{I}_{3}
$$

where

$$
\mathcal{I}_{3} \triangleq \sum_{j=0}^{\infty} \frac{K_{2}^{j}}{j !} \sum_{k=1}^{j+1} E_{k}\left(\frac{c\left(1+K_{2}\right) N_{2}}{\Omega_{2} P_{2}}\right) .
$$

Partitioning $\mathcal{I}_{3}$ into two parts, we can bound $\mathcal{I}_{3}$ as

$$
\mathcal{I}_{3}<\sum_{j=0}^{N-1} \frac{K_{2}^{j}}{j !} \sum_{k=1}^{j+1} E_{k}\left(\frac{c\left(1+K_{2}\right) N_{2}}{\Omega_{2} P_{2}}\right)+\mathcal{I}_{4}
$$

where

$$
\mathcal{I}_{4} \triangleq E_{1}\left(\frac{c\left(1+K_{2}\right) N_{2}}{\Omega_{2} P_{2}}\right) \sum_{j=N}^{\infty} \frac{K_{2}^{j}(j+1)}{j !} .
$$

Note that the inequality in (45) stems from the fact that $E_{n}(x)$ is a monotonically decreasing function of $n$. Now, $\mathcal{I}_{4}$ can explicitly be computed as

$$
\begin{aligned}
\mathcal{I}_{4}=E_{1} & \left(\frac{c\left(1+K_{2}\right) N_{2}}{\Omega_{2} P_{2}}\right) \exp \left(K_{2}\right) \\
& \times\left(\frac{K_{2}(N-1) \gamma\left(N-1, K_{2}\right)+\gamma\left(N, K_{2}\right)}{\Gamma(N)}\right) .
\end{aligned}
$$

We note that (47) stems from the power series expansion of $\gamma(p, x)$ [19, Eq. (6.5.29)]. The final observation is that $\log _{2}(1+$ $a \exp (x))$ is an increasing function of $x$ for $a>0$.

\section{APPENDIX D}

\section{ProOF OF PROPOSITION 8}

The key task is to compute $\mathrm{E}\left\{\left|h_{i}\right|^{2}\right\}$ and $\mathrm{E}\left\{\left|h_{i}\right|^{2} /\left(\left|h_{i}\right|^{2}+\right.\right.$ a) $\}$. The first term can trivially be obtained from the definition of the Rician pdf according to

$$
\mathrm{E}\left\{\left|h_{i}\right|^{2}\right\}=\Omega_{i} .
$$

The second term can be evaluated as

$\mathrm{E}\left\{\frac{\left|h_{i}\right|^{2}}{\left|h_{i}\right|^{2}+a}\right\}=\frac{\left(1+K_{i}\right)}{\Omega_{i} e^{K_{i}}} \sum_{k=0}^{\infty} \frac{1}{(k !)^{2}}\left(\frac{K_{i}\left(K_{i}+1\right)}{\Omega_{i}}\right)^{k} \mathcal{I}_{5}$

where

$$
\mathcal{I}_{5} \triangleq \int_{0}^{\infty} \frac{x^{k+1}}{x+a} \exp \left(-\frac{\left(1+K_{i}\right) x}{\Omega_{i}}\right) d x
$$

which can explicitly be evaluated as

$$
\begin{aligned}
& \mathcal{I}_{5}=\exp \left(\frac{\left(1+K_{i}\right) a}{\Omega_{i}}\right)\left(\frac{1+K_{i}}{\Omega_{i}}\right)^{-k-1} \\
& \times E_{k+2}\left(\frac{\left(1+K_{i}\right) a}{\Omega_{i}}\right) \Gamma(k+2) .
\end{aligned}
$$

Now, substituting (51) into (49), we have

$$
\mathrm{E}\left\{\frac{\left|h_{i}\right|^{2}}{\left|h_{i}\right|^{2}+a}\right\}=\exp \left(\frac{\left(1+K_{i}\right) a}{\Omega_{i}}-K_{i}\right) \mathcal{I}_{6}
$$

where

$$
\mathcal{I}_{6} \triangleq \sum_{k=0}^{\infty} \frac{(k+1) K_{i}^{k}}{k !} E_{k+2}\left(\frac{\left(1+K_{i}\right) a}{\Omega_{i}}\right) .
$$

Splitting the infinity summation into two parts, we can bound $\mathcal{I}_{6}$ as

$$
\begin{aligned}
\mathcal{I}_{6}<\sum_{k=0}^{N-1} \frac{(k+1) K_{i}^{k}}{k !} & E_{k+2}\left(\frac{\left(1+K_{i}\right) a}{\Omega_{i}}\right) \\
& +E_{N+2}\left(\frac{\left(1+K_{i}\right) a}{\Omega_{i}}\right) \sum_{k=N}^{\infty} \frac{(k+1) K_{i}^{k}}{k !} .
\end{aligned}
$$

Utilizing the series representation of $\gamma(p, x),(54)$ can be solved in closed form. The desired result then follows from the fact that $\log _{2}(1+a x)$ is an increasing function with respect to $x$ for $a>0$. 


\section{REFERENCES}

[1] J. N. Laneman, D. N. C. Tse, and G. W. Wornell, "Cooperative diversity in wireless networks efficient protocols and outage behavior," IEEE Trans. Inf. Theory, vol. 50, no. 12, pp. 3062-3080, Dec. 2004.

[2] M. O. Hasna and M.-S. Alouini, "End-to-end performance of transmission systems with relays over Rayleigh-fading channels," IEEE Trans. Wireless Commun., vol. 2, no. 6, pp. 1126-1131, Nov. 2003.

[3] M. O. Hasna and M.-S. Alouini, "A performance study of dual-hop transmissions with fixed gain relays," IEEE Trans. Wireless Commun., vol. 3 , no. 6, pp. 1963-1968, Nov. 2004.

[4] G. Farhadi and N. C. Beaulieu, "On the ergodic capacity of wireless relaying systems over Rayleigh fading channels," IEEE Trans. Wireless Commun., vol. 7, no. 11, pp. 4462-4467, Nov. 2008.

[5] G. Farhadi and N. C. Beaulieu, "On the ergodic capacity of multihop wireless relaying systems," IEEE Trans. Wireless Commun., vol. 8, no. 5, pp. 2286-2291, May 2009.

[6] O. Waqar, M. Ghogho, and D. McLernon, "Tight bounds for ergodic capacity of dual-hop fixed-gain relay networks under Rayleigh fading," IEEE Commun. Lett., vol. 15, no. 4, pp. 413-415, Apr. 2011.

[7] D. B. da Costa and S. Aïssa, "Capacity analysis of cooperative systems with relay selection in Nakagami- $m$ fading," IEEE Commun. Lett., vol. 13, no. 9, pp. 637-639, Sep. 2009.

[8] S. Ikki and M. H. Ahmed, "Performance analysis of dual-hop relaying over non-identical Weibull fading channels," in Proc. IEEE VTC, Barcelona, Spain, Apr. 2009, pp. 1-5.

[9] S. Ikki and M. H. Ahmed, "Performance analysis of cooperative diversity wireless networks over Nakagami- $m$ fading channel," IEEE Commun. Lett., vol. 11, no. 4, pp. 334-336, Apr. 2007.

[10] L. Wu, K. Niu, Z. He, W. Xu, and J. Lin, "Ergodic capacity of dual-hop transmissions over composite multipath/shadowing channels," Electron. Lett., vol. 45, no. 19, pp. 975-976, Sep. 2009.

[11] O. Waqar, D. McLernon, and M. Ghogho, "Exact evaluation of ergodic capacity for multihop variable-gain relay networks: A unified framework for generalized fading channels," IEEE Trans. Veh. Technol., vol. 59, no. 8, pp. 4181-4187, Oct. 2010.

[12] M. Di Renzo, F. Graziosi, and F. Santucci, "Channel capacity over generalized fading channels: A novel MGF-based approach for performance analysis and design of wireless communication systems," IEEE Trans. Veh. Technol., vol. 59, no. 1, pp. 127-149, Jan. 2010

[13] W. W. Piegorsch and G. Casella, "The existence of the first negative moment," Amer. Statistician, vol. 39, no. 1, pp. 60-62, Feb. 1985.

[14] M. Nakagami, "The $m$-distribution-A general formula of intensity distribution of rapid fading," in Statistical Methods in Radio Wave Propagation, W. C. Hoffman, Ed. Oxford, U.K.: Pergamon, 1960, pp. 3-36.

[15] H. Suzuki, "A statistical model for urban multipath propagation," IEEE Trans. Commun., vol. 25, no. 7, pp. 673-680, Jul. 1977.

[16] A. U. Sheikh, M. Handforth, and M. Abdi, "Indoor mobile radio channel at $946 \mathrm{MHz}$ : Measurements and modeling," in Proc. IEEE VTC, Secaucus, NJ, May 1993, pp. 73-76.

[17] M. Matthaiou, D. I. Laurenson, and J. S. Thompson, "A MIMO channel model based on the Nakagami-faded spatial eigenmodes," IEEE Trans. Antennas Propag., vol. 56, no. 5, pp. 1494-1497, May 2008.

[18] I. S. Gradshteyn and I. M. Ryzhik, Table of Integrals, Series, and Products, 5th ed. Orlando, FL: Academic, 1994.

[19] M. Abramowitz and I. A. Stegun, Handbook of Mathematical Functions. New York: Dover, 1974.

[20] H. Shin and J. Lee, "Capacity of multiple-antenna fading channels: Spatial fading correlation, double scattering, and keyhole," IEEE Trans. Inf. Theory, vol. 49, no. 10, pp. 2636-2647, Oct. 2003.

[21] D. B. da Costa and M. D. Yacoub, "Dual-hop transmissions with semiblind relays over Nakagami- $m$ fading channel," Electron. Lett., vol. 44, no. 3, pp. 214-216, Jan. 2008.

[22] F. Babich and G. Lombardi, "Statistical analysis and characterization of the indoor propagation channel," IEEE Trans. Commun., vol. 48, no. 3, pp. 455-464, Mar. 2000.

[23] G. Tzeremes and C. G. Christodoulou, "Use of Weibull distribution for describing outdoor multipath fading," in Proc. Antennas Propag. Soc. Int. Symp., San Antonio, TX, Jun. 2002, vol. 1, pp. 232-235.

[24] N. C. Sagias, D. A. Zogas, G. K. Karagiannidis, and G. S. Tombras, "Channel capacity and second-order statistics in Weibull fading," IEEE Commun. Lett., vol. 8, no. 6, pp. 377-379, Jun. 2004.

[25] K. A. Stewart, G. P. Labedz, and K. Sohrabi, "Wideband channel measurements at $900 \mathrm{MHz}$," in Proc. IEEE VTC, Chicago, IL, Jul. 1995, pp. 236-240.

[26] R. Bultitude, S. A. Mahmoud, and W. A. Sullivan, "A comparison of indoor radio propagation characteristics at $910 \mathrm{MHz}$ and $1.75 \mathrm{GHz}$," IEEE J. Sel. Areas Commun., vol. 7, no. 1, pp. 20-30, Jan. 1989.
[27] P. M. Shankar, "Error rates in generalized shadowed fading channels," Wireless Pers. Commun., vol. 28, no. 3, pp. 233-238, Feb. 2004.

[28] P. S. Bithas, N. C. Sagias, P. T. Mathiopoulos, G. K. Karagiannidis, and A. A. Rontogiannis, "On the performance analysis of digital communications over generalized- $K$ fading channels," IEEE Commun. Lett., vol. 10 no. 5, pp. 353-355, May 2006.

[29] I. M. Kostic, "Analytical approach to performance analysis for channel subject to shadowing and fading," Proc. Inst. Elect. Eng.-Commun., vol. 152, no. 6, pp. 821-827, Dec. 2005.

[30] A. Laourine, M.-S. Alouini, S. Affes, and A. Stéphenne, "On the capacity of generalized- $K$ fading channels," IEEE Trans. Wireless Commun., vol. 7, no. 7, pp. 2441-2445, Jul. 2008.

[31] M. Matthaiou, N. D. Chatzidiamantis, G. K. Karagiannidis, and J. A. Nossek, "On the capacity of generalized- $K$ fading MIMO channels," IEEE Trans. Signal Process., vol. 58, no. 11, pp. 5939-5944, Nov. 2010.

[32] A. P. Prudnikov, Y. A. Brychkov, and O. I. Marichev, Integrals and Series: Special Functions. New York: Taylor \& Francis, 1998.

[33] H. A. Suraweera, G. K. Karagiannidis, and P. J. Smith, "Performance analysis of the dual-hop asymmetric fading channel," IEEE Trans. Wireless Commun., vol. 8, no. 6, pp. 2783-2788, Jun. 2009.

[34] A. P. Prudnikov, Y. A. Brychkov, and O. I. Marichev, Integrals and Series, Volume 3: More Special Functions. New York: Gordon and Breach, 1990.

[35] A. M. Mathai and R. K. Saxena, Generalized Hypergeometric Functions With Applications in Statistics and Physical Sciences. Berlin, Germany: Springer-Verlag, 1973.

[36] V. S. Adamchik and O. I. Marichev, "The algorithm for calculating integrals of hypergeometric type functions and its realization in REDUCE system," in Proc. Int. Conf. Symbolic Algebraic Comput., Tokyo, Japan, 1990, pp. 212-224.

[37] A. Lapidoth and S. M. Moser, "Capacity bounds via duality with applications to multiple-antenna systems on the flat-fading channels," IEEE Trans. Inf. Theory, vol. 49, no. 10, pp. 2426-2467, Oct. 2003.

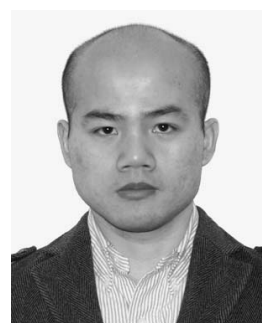

Caijun Zhong (S'07-M'10) received the B.S. degree in information engineering from Xi'an Jiaotong University, Xi' an, China, in 2004 and the M.S. degree in information security and the Ph.D. degree in telecommunications from University $\mathrm{Col}-$ lege London, London, U.K., in 2006 and 2010, respectively.

From September 2009 to September 2011, he was a Research Fellow with the Institute for Electronics, Communications and Information Technologies, Queen's University Belfast, Belfast, U.K. Since October 2011, he has been an Assistant Professor with Zhejiang University, Hangzhou, China. His research interests include multivariate statistical theory, mutliple-input-multiple-output communications systems, cooperative communications, and cognitive radio systems.

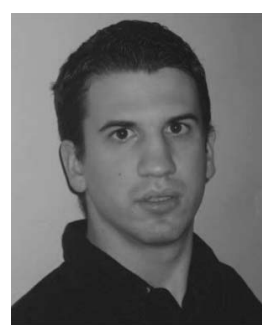

Michail Matthaiou (S'05-M'08) was born in Thessaloniki, Greece, in 1981. He received the Diploma degree (five years) in electrical and computer engineering from the Aristotle University of Thessaloniki, in 2004, the M.Sc. degree (with distinction) in communication systems and signal processing from the University of Bristol, Bristol, U.K., in 2005, and the Ph.D. degree from the University of Edinburgh, Edinburgh, U.K., in 2008.

From September 2008 through May 2010, he was a Postdoctoral Research Associate with the Institute for Circuit Theory and Signal Processing, Munich University of Technology, Munich, Germany. In June 2010, he joined Chalmers University of Technology, Gothenburg, Sweden, as an Assistant Professor, and in 2011, he was awarded the Docent title. His research interests span signal processing for wireless communications, random matrix theory and multivariate statistics for mutlipleinput-multiple-output systems, and performance analysis of fading channels.

Dr. Matthaiou co-received the 2006 IEEE Communications Chapter Project Prize for the best M.Sc. dissertation in the area of communications and has been an Exemplary Reviewer for IEEE COMMUNICATIONS LETTERS for 2010. He has been a member of Technical Program Committees for several IEEE conferences, such as the Global Communications Conference, Digital Signal Processing, etc. He currently serves as an Associate Editor for IEEE COMMUNiCATIONS LeTtERs and as a Lead Guest Editor of the special issue on "Large-scale multiple antenna wireless systems" of the IEEE JOURNAL ON SElected AREas in Communications. He is an associate member of the IEEE Signal Processing Society SPCOM technical committee. 


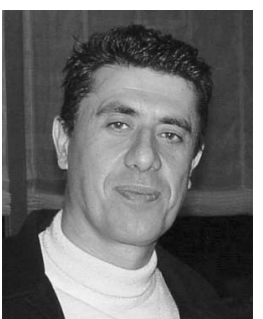

George K. Karagiannidis (M'97-SM'04) was born in Pithagorion, Samos Island, Greece. He received the University Diploma (five years) and Ph.D. degrees in both electrical and computer engineering from the University of Patras, Patras, Greece, in 1987 and 1999 , respectively.

From 2000 to 2004, he was a Senior Researcher with the Institute for Space Applications and Remote Sensing, National Observatory of Athens, Athens, Greece. In June 2004, he joined Aristotle University of Thessaloniki, Thessaloniki, Greece, where he is currently an Associate Professor of digital communications systems with the Electrical and Computer Engineering Department and the Head of the Telecommunications Systems and Networks Lab. He is the author or coauthor of more than 120 technical papers published in scientific journals and presented at international conferences. He is also a coauthor of three chapters in books and the author of the Greek edition book Telecommunications Systems. His current research interests are in the broad area of digital communications systems with emphasis on cooperative communication, adaptive modulation, mutliple-input-multiple-output systems, optical wireless, and underwater communications.

Dr. Karagiannidis has been a member of the Technical Program Committees for several IEEE conferences such as the International Conference on Communications (ICC), Global Communications Conference, etc. He is a member of the editorial boards of the IEEE TRANSACTIONS ON COMMUNICATIONS, Senior Editor of the IEEE COMMUNiCATIONS LETTERS, and Lead Guest Editor of the special issue on "Optical Wireless Communications" of the IEEE Journal on SElected AREAS on Communications. He co-received the Best Paper Award from the Wireless Communications Symposium at the IEEE ICC'07, Glasgow, U.K., June 2007. He is the Chair of the IEEE Communications Society Greek Chapter.

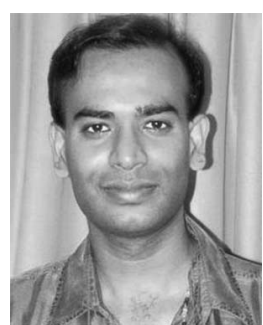

Tharmalingam Ratnarajah (A'96-M'05-SM'05) received the B.Eng. (Honors), M.Sc., and Ph.D. degrees.

Since 1993, he has held various research positions with the University of Ottawa, Ottawa, ON, Canada; Nortel Networks, Ottawa; McMaster University, Hamilton, ON; and Imperial College, London, U.K. He is currently with the Institute for Electronics, Communications, and Information Technologies, Queen's University Belfast, Belfast, U.K. He has published over 130 publications in these areas and holds four U.S. patents. He is currently the coordinator of the FP7 Future and Emerging Technologies projects "CROWN" in the area of cognitive radio networks and "HIATUS" in the area of interference alignment. His research interests include random matrix theory, information theoretic aspects of mutliple-input-multiple-output channels and ad hoc networks, wireless communications, signal processing for communication, statistical and array signal processing, biomedical signal processing, and quantum information theory.

Dr. Ratnarajah is a member of the American Mathematical Society and the Information Theory Society. 